\title{
Propuesta para manejo de la coagulopatía asociada a la COVID-19 en adultos
}

\author{
Gregorio Ignacio-Ibarra, ${ }^{1}$ Ma. Teresa García-Lee, ${ }^{1}$ Ana I. González-Ávila, ${ }^{1}$ Jaime García-Chávez, ${ }^{2}$ \\ Laura Guzmán-Chores, ${ }^{1}$ Cindy Ledesma-de la Cruz, ${ }^{1}$ Yolanda Lugo-García, ${ }^{1}$ Jessi Juárez-Lara, ${ }^{1}$ \\ Mauricio García-Vázquez, ${ }^{1}$ Cristina E. Madera-Maldonado, ${ }^{3}$ Christian O. Ramos-Peñafie ${ }^{3}$ y \\ Abraham Majluf-Cruz',4*
}

${ }^{1}$ Servicio de Hematología, Hospital General Regional N. 1 Carlos MacGregor Sánchez Navarro, Instituto Mexicano del Seguro Social, Ciudad de México; ${ }^{2}$ Servicio de Hematología, Unidad Médica de Alta Especialidad, Hospital de Especialidades Eduardo Fraga Mouret, Centro Médico Nacional La Raza, Instituto Mexicano del Seguro Social, Ciudad de México; ${ }^{3}$ Servicio de Hematología, Hospital Regional de Alta Especialidad de Ixtapaluca, Secretaría de Salud, Ixtapaluca, Edo. Méx.; ${ }^{4}$ Unidad de Investigación Médica en Trombosis, Hemostasia y Aterogénesis, Hospital General Regional N. 1 Carlos MacGregor Sánchez Navarro, Instituto Mexicano del Seguro Social, Ciudad de México. México

\section{Resumen}

La infección por coronavirus 2 del síndrome respiratorio agudo grave (SARS-CoV-2) es la causante de la pandemia de enfermedad por coronavirus 2019 (COVID-19), con un índice de letalidad alto. La mayoría de los pacientes graves desarrollan un tipo especial de coagulopatía no descrito hasta ahora y la cual se considera ahora la principal causa de muerte. Por esta razón, el tratamiento anticoagulante se ha convertido en una de las piedras angulares del tratamiento de esta infección. Sin embargo, la velocidad con la que se genera la evidencia respecto al uso de anticoagulantes es muy rápida y, en ocasiones difícil de interpretar y contradictoria. Luego de hacer una revisión extensa de la literatura publicada, se hace esta propuesta para el uso del tratamiento anticoagulante tomando en cuenta los recursos disponibles en México.

PALABRAS CLAVE: COVID-19. Coagulopatía. Coagulación intravascular diseminada. Anticoagulantes. Enfermedad tromboembólica venosa.

\section{A proposal for the management of COVID-19-induced coagulopathy in adults}

\begin{abstract}
Severe acute respiratory syndrome coronavirus 2 (SARS-CoV-2) infection is the cause of the coronavirus disease 2019 (COVID-19) pandemic, which has a high case fatality rate. Most severely ill patients develop a special type of coagulopathy that had not been described before and that is now considered the main cause of death. For this reason, anticoagulant treatment has become one of the cornerstones of the treatment of this infection. However, the rate at which the evidence regarding the use of anticoagulants is generated is quite fast, and sometimes it is difficult to interpret and conflicting. After having performed an extensive review of the published literature, this proposal for the use of anticoagulant treatment is made, taking into account available resources in Mexico.
\end{abstract}

KEY WORDS: COVID-19. Coagulopathy Disseminated intravascular coagulation. Anticoagulants Venous thromboembolic disease.

Correspondencia:

*Abraham Majluf-Cruz

E-mail: amajlufc@gmail.com
Fecha de recepción: 12-12-2020

Fecha de aceptación: 17-12-2020

DOI: $10.24875 / G M M .20000930$
Gac Med Mex. 2021;157:209-214

Disponible en PubMed

www.gacetamedicademexico.com CC BY-NC-ND (http://creativecommons.org/licenses/by-nc-nd/4.0/). 


\section{Introducción}

La enfermedad por coronavirus 2019 (COVID-19), causada por el coronavirus 2 del síndrome respiratorio agudo grave (SARS-CoV-2), se identificó por primera vez en diciembre de 2019 en Wuhan (China) y es reconocida hoy como una pandemia por la Organización Mundial de la Salud. La infección por este virus predispone a la enfermedad trombótica, esencialmente venosa, debido a una respuesta inflamatoria excesiva, hipoxia, inmovilización, ventilación asistida y un tipo nuevo de coagulación intravascular diseminada, esta última un tanto diferente de la forma clásica. ${ }^{1-4}$ Así, las complicaciones tromboembólicas se convirtieron en la principal causa de muerte asociada a esta infección viral. ${ }^{1-5}$ La infección ha contribuido significativamente a la mortalidad en una gran cantidad de países, con un aumento exponencial de los casos en todo el mundo. ${ }^{5}$ La mayoría de la población mundial será afectada levemente por la infección, pero los sujetos que pasen a la forma más agresiva sufrirán una forma de afección monoorgánica de la enfermedad caracterizada por insuficiencia respiratoria. En las formas más graves, sin embargo, la enfermedad progresará hasta una forma más sistémica caracterizada por disfunción orgánica múltiple. Todo indica que uno de los mecanismos más importantes para llegar al estado grave y que al mismo tiempo es uno de los marcadores pronóstico más graves para llegar a la muerte es la coagulopatía asociada a COVID-19. ${ }^{2}$

Debido a esta coagulopatía, es necesario describir dos escenarios importantes asociados a ella. Primero, la presencia de una coagulopatía similar a la de una coagulación intravascular diseminada tradicional (estado hipercoagulable), la cual parece responsable en gran medida del desarrollo último de la falla orgánica múltiple que complica a esta infección. Este estado hipercoagulable sistémico puede demostrarse por los resultados de las pruebas de laboratorio disponibles: trombocitopenia, alargamiento del tiempo de protrombina y del tiempo parcial de tromboplastina parcial activada (TTPa), aumento del dímero D y de los productos de degradación de la fibrina y disminución de la concentración del fibrinógeno, entre otras. Segundo, la aparición de eventos tromboembólicos, fundamentalmente venosos (trombosis venosa profunda y tromboembolia pulmonar), que cada vez más se diagnostican en el paciente infectado grave. Debido a que los pacientes podían presentarse con trombocitopenia ocasionalmente y con alargamiento de los tiempos de coagulación la primera respuesta fue evitar el tratamiento anticoagulante incluso de manera profiláctica. Hoy sabemos que los anticoagulantes se erigen como una de las piedras angulares del tratamiento del paciente infectado grave. Por otra parte, un marcador de activación hemostásica, el dímero D, es el mejor marcador pronóstico con el que contamos en este momento para la toma de decisiones para el paciente. ${ }^{6}$

Como ya se mencionó, aunque en el principio de la enfermedad el uso de anticoagulantes se evitó sistemáticamente, tener en este momento una idea clara del problema hemostásico y su manera de abordarlo es imperativo para la atención integral del paciente haciendo énfasis en que no solo se trata de un problema de la microvasculatura inductora de falla orgánica múltiple, sino también de complicaciones macrotrombóticas venosas, las cuales complican más aún el escenario clínico de estos enfermos, tanto en los servicios de urgencias, los pisos generales y en las unidades de cuidados intensivos. Debido a todo esto y a la proclividad de la infección a asociarse con eventos trombóticos venosos, las guías internacionales sugieren fuertemente la evaluación de los factores de riesgo para enfermedad tromboembólica venosa en todo paciente con COVID-19, ya que su presencia eleva significativamente la posibilidad de complicaciones trombóticas venosas y empobrece significativamente el pronóstico del enfermo.

El objetivo de este este material es brindar al médico a cargo de pacientes con COVID-19 sugerencias claras y precisas basadas en la literatura acerca del manejo anticoagulante para estos enfermos.

\section{Material y métodos}

Hicimos una búsqueda dirigida de la información publicada en las últimas semanas en PubMed. Se hizo el análisis de la gran mayoría de ellas y evaluamos cuáles tenían información aplicable a los recursos disponibles en el Sector Salud México. Consideramos dos aspectos fundamentales. Primero, como prevenir o revertir la coagulopatía asociada a COVID-19. Segundo, cuáles medidas tomar en relación con la hemorragia asociada al tratamiento anticoagulante. Las recomendaciones de este trabajo tienen su base en una serie de publicaciones, las cuales se encuentran disponibles en la literatura y pueden ser consultadas de manera gratuita. ${ }^{7-24}$ Por último, las recomendaciones descritas han sido 


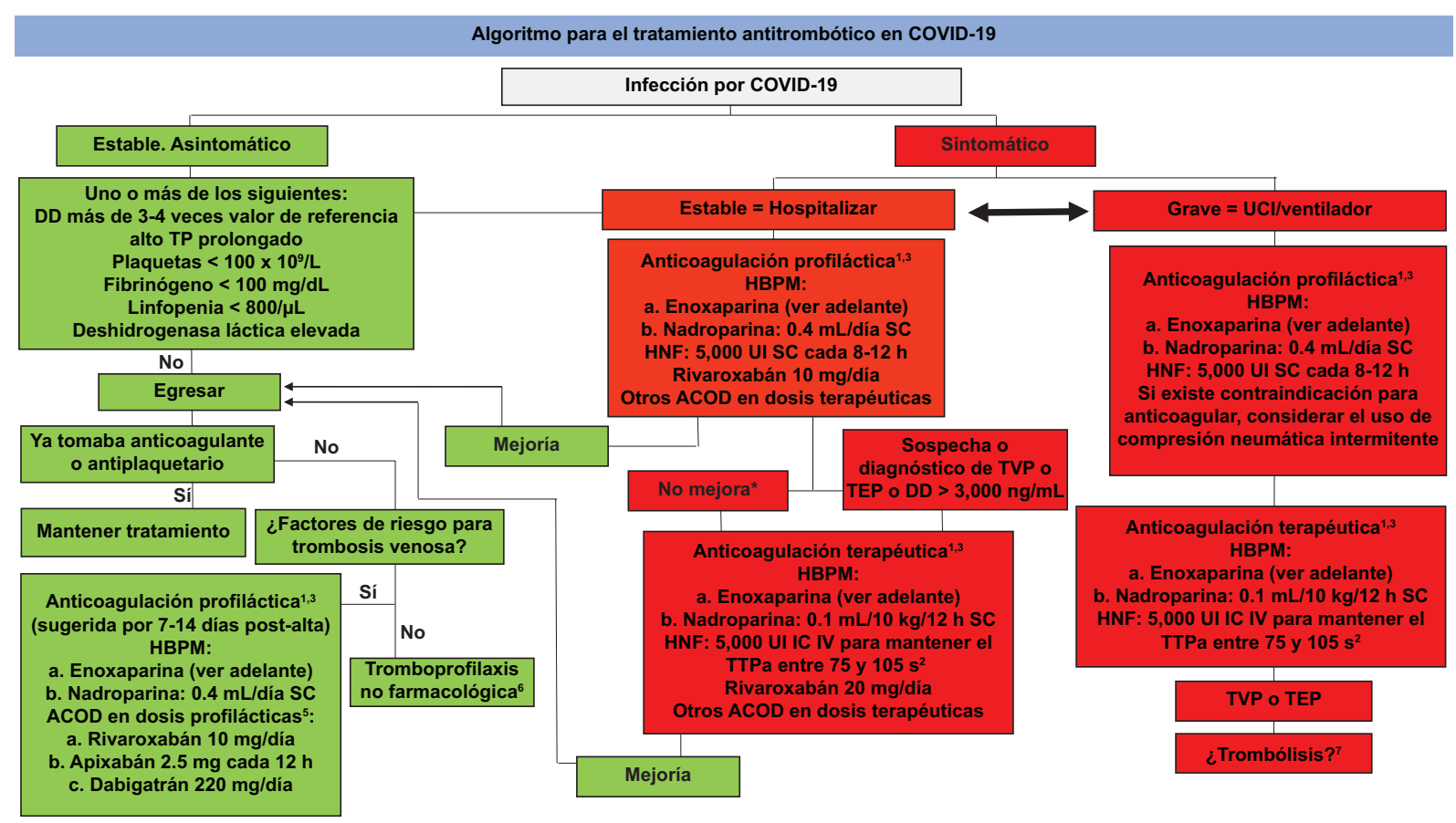

Figura 1. Algoritmo propuesto para el uso del tratamiento anticoagulante en los pacientes con enfermedad por coronavirus 2019 (COVID-19). Se incluyen algunas sugerencias para la decisión de hospitalizar o no al paciente. $D D=$ dímero $D ; T P=$ tiempo de protrombina; TTPa $=$ tiempo de tromboplastina parcial activada; HBPM = heparina de bajo peso molecular; HNF = heparina no fraccionada; $A C O D=$ anticoagulantes orales directos; $S C$ = subcutánea; $I V=$ intravenosa; IC = infusión continua; TVP = trombosis venosa profunda; TEP = tromboembolia pulmonar. ${ }^{*}$ En caso de no mejoría y sí el DD se encuentra entre 1,000-3,000 ng/mL se recomienda profilaxis con dosis intermedia (ver adelante). Ver definiciones de los superíndices en la Figura 2.

aderezadas con la experiencia ganada en los últimos meses con el tratamiento antitrombótico en los pacientes con COVID-19.

\section{Resultados}

Del análisis de la literatura publicada, realizamos las Figuras 1-3. Las primeras dos se relacionan con el primer objetivo de las recomendaciones: prevenir o revertir la coagulopatía. La Figura 3 es un intento resumido y práctico para el tratamiento de la hemorragia asociada al tratamiento anticoagulante.

\section{Discusión}

Desde el inicio de su aparición hace unos meses, la infección por SARS-CoV-2 se caracterizó por asociarse a una mortalidad alta. Inmediatamente se observó que en la gran mayoría de los enfermos existía un estado de activación descontrolada del sistema hemostásico. A este estado patológico se le identificó como una coagulación intravascular diseminada clásica. Sin embargo, al pasar las semanas, se evidenció que esta coagulopatía no cumplía las características aceptadas para la coagulación intravascular diseminada, por lo que en la actualidad se considera como una variante del concepto clásico y se define mejor como coagulopatía asociada a COVID-19. Esta se presenta con una elevación importante del dímero $\mathrm{D}$ y de los productos de degradación de fibrina/fibrinógeno, mientras que otras alteraciones de los parámetros de la coagulación (TTPa y plaquetas, entre otras) son poco frecuentes al inicio de la enfermedad. Del conocimiento de la coagulopatía asociada a COVID-19 han derivado acuerdos generales que se aceptan ya en el mundo y que han sido emitidos en diversas recomendaciones de guías internacionales: a) todos los pacientes hospitalizados por COVID-19 deben recibir tromboprofilaxis; b) se debe evaluar la profilaxis antitrombótica en todos los pacientes con COVID-19 con factores de riesgo de trombosis, y c) el mejor esquema de anticoagulación no está aún definido completamente, ya que todos los días se hacen aportes nuevos al conocimiento en este sentido.

Hoy tenemos claro que el tratamiento para la infección para este virus incluirá las siguientes estrategias: 1) medicamentos antivirales; 2) moduladores de la 


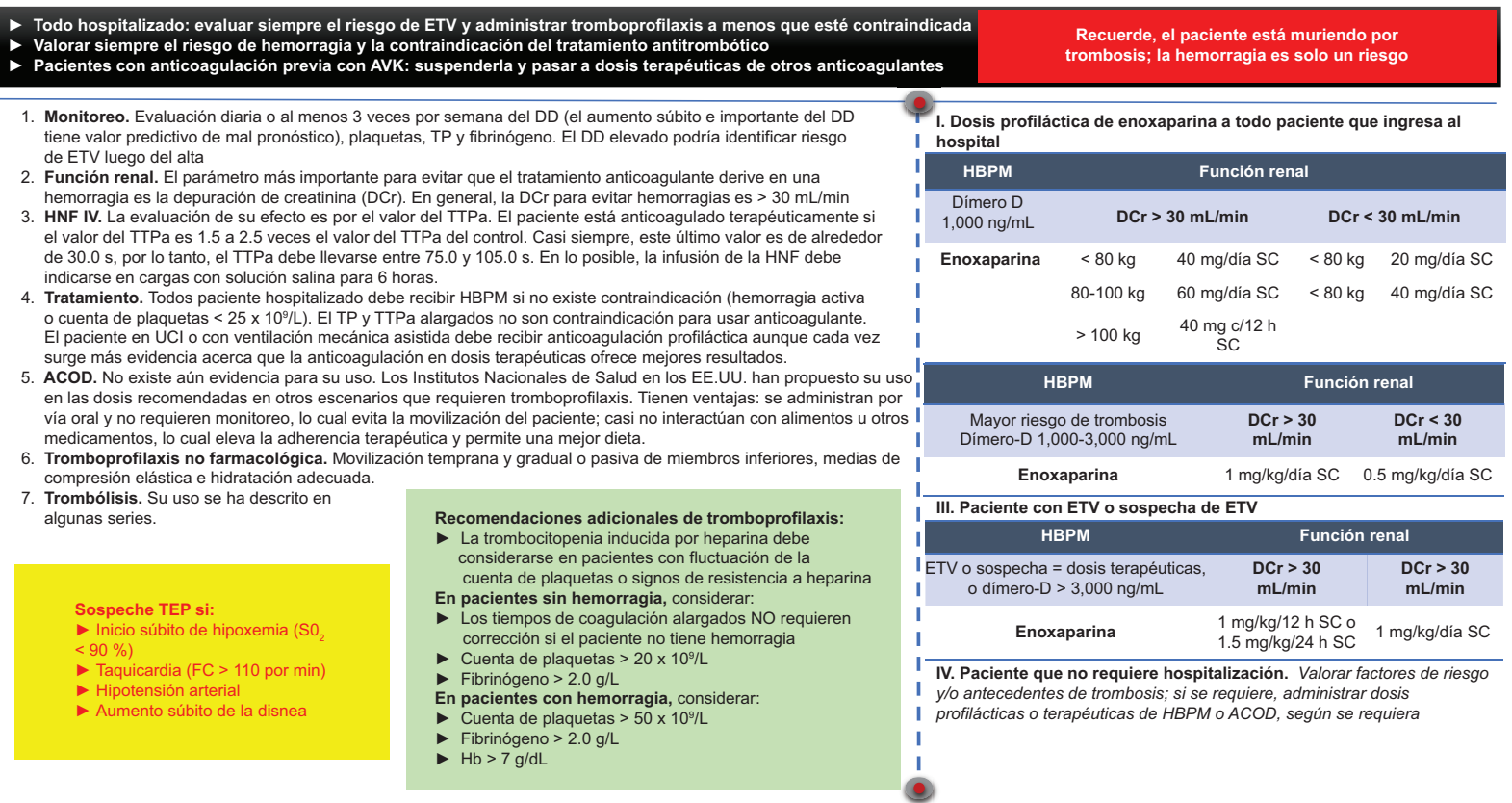

Figura 2. Recomendaciones hematológicas para el tratamiento antitrombótico en COVID-19. Se muestran algunos puntos importantes incluyendo la definición de los superíndices utilizados en la Figura 1. AVK = anticoagulantes antagonistas de la vitamina K (acenocumarina y warfarina); $E T V=$ enfermedad tromboembólica venosa; $D D=$ dímero $D ; T P=$ tiempo de protrombina; TTPa = tiempo de tromboplastina parcial activada; $H B P M=$ heparina de bajo peso molecular; $H N F=$ heparina no fraccionada; $A C O D=$ anticoagulantes orales directos; $S C=$ subcutánea; IV = intravenosa; $F C=$ frecuencia cardiaca; $H b=$ hemoglobina .

Recomendaciones hematológicas para el manejo de la hemorragia asociada al tratamiento antitrombótico en
COVID-19-8,10-14,17,18,20
ABC para el abordaje de la hemorragia:
A. Analizar: chequear signos vitales, analizar la función renal, función hepática, tratamiento concomitante,
hidratación del paciente, estudios de laboratorio
B. Buscar: sitio de la hemorragia, magnitud de la hemorragia (la hemorragia es mayor o no mayor)
C. Controlar

- Tratamiento estándar para el control de la hemorragia:

- Considere el tratamiento sintomático adecuado

- Compresión mecánica, siempre que sea posible

- Corregir la hipovolemia con reposición de líquido y/o apoyo hemodinámico

- Corregir acidosis, hipoxia e hipotermia

- Revalorar la función renal, si la $\mathrm{DCr}<30 \mathrm{~mL} / \mathrm{min}$, revisar y ajustar las dosis de la heparina

- Transfusión de productos o componentes sanguíneos en pacientes con hemorragia

- Si la hemoglobina es $\leq 7 \mathrm{~g} / \mathrm{dL}$ transfundir 1 concentrado eritrocitario y posteriormente revalorar

- Si la cuenta de plaquetas es $\leq 20 \times 10^{9} / \mathrm{L}$ transfundir concentrados plaquetarios y revalorar

- Si los tiempos de coagulación están prolongados administrar plasma fresco congelado y revalorar

- Si el nivel de fibrinógeno es $<100 \mathrm{mg} / \mathrm{mL}$ transfundir crioprecipitados

- Ácido tranexámico: usar solo si no hay contraindicación. No indicado en presencia de CID

- rFVlla no recomendado

Figura 3. Recomendaciones hematológicas para el manejo de la hemorragia asociada a la tromboprofilaxis en enfermedad por coronavirus 2019 (COVID-19). Aspectos que tomar en cuenta en el caso del paciente con hemorragia secundaria al uso de anticoagulantes. CID =coagulación intravascular diseminada; rFVIla = factor VII activado recombinante; $\mathrm{DCr}=$ depuración de creatinina.

respuesta inflamatoria; 3) moduladores de la invasión viral, y 4) control de la coagulopatía asociada al virus. A diferencia de lo que ocurre con las tres primeras estrategias, hoy existe mucho más acuerdo y evidencia acerca del tratamiento que seguir con la coagulopatía, aunque todos los días se hacen aportes nuevos al conocimiento en este sentido.

Se debe aclarar que, como ya se ha publicado en diferentes revistas, existe el riesgo de escasez de heparinas en el mundo. Esto es algo que considerar 
si se recuerda que las heparinas no fraccionadas y las de bajo peso molecular son biofármacos obtenidos de tejidos no humanos, procesados y elaborados bajo normas estrictas de control de calidad. Por esta razón, los anticoagulantes orales directos rivaroxabán y betrixabán han sido recomendados por los National Institutes of Health para su uso en el paciente que es egresado del hospital. Ya que en México en este momento solo contamos con rivaroxabán, se hace la referencia a esta recomendación, sin embargo, es posible que los otros dos anticoagulantes directos disponibles en nuestro país, apixabán y dabigatrán, pudieran ser empleados también en las dosis empleadas en la tromboprofilaxis de la cirugía ortopédica mayor.

Esta es una guía para el manejo de la coagulopatía y la enfermedad tromboembólica venosa en el paciente con COVID-19. Debemos subrayar que el contenido propuesto no es definitivo, cambia semana a semana, por lo que el médico requiere mantenerse actualizado en todo momento acerca de este problema. Esta guía fue elaborada tomando en consideración los recursos actuales del sistema de salud público mexicano (los cuales no son pocos). Como toda guía, es solo la compilación de sugerencias publicadas en la literatura y, por lo tanto, de ningún modo puede sustituir al juicio clínico, a la experiencia médica y a las consideraciones tomadas al lado del paciente considerando las necesidades de cada enfermo y los recursos con que cuente el médico en ese momento. Por supuesto, esta información no sustituye al criterio médico.

El objetivo de generar esta guía fue apoyar a los médicos del sector salud ( $y$, quizá, de otras instituciones), para la toma de decisiones más rápida en caso de que se encuentren tratando pacientes con COVID-19. Las complicaciones trombóticas asociadas a esta infección viral se reconocen ya como la principal causa final de muerte en estos pacientes y resaltan la importancia del sistema de la coagulación en la medicina actual. Esta guía no contempla los eventos trombóticos arteriales, ya que, debido a la naturaleza de la coagulopatía y como se reconoce cada vez más en otros ámbitos de le medicina moderna, el impacto más grande es en el sistema venoso. La gravedad de la coagulopatía y la velocidad con la que se están evidenciando los fenómenos trombóticos hace que se estén liberado los artículos por vía electrónica. Por supuesto, el lector debe considerar dos cosas importantes. Primero, las posibilidades terapéuticas cambian en cualquier momento, por lo cual se sugiere encarecidamente al lector que siga revisando la literatura generada. Segundo, la lectura de la información que se está generando debe ser muy crítica; hay una gran cantidad de publicaciones sin el rigor científico suficiente para considerarlas seriamente.

\section{Conclusiones}

La coagulopatía asociada a COVID-19 es considerada la complicación más importante de la infección por el SARS-CoV-2 y quizá la principal causa de muerte entre los enfermos afectados. Por lo tanto, el tratamiento antitrombótico, tanto en sus variedades profilácticas o terapéuticas, se ha convertido en una de las piedras angulares del tratamiento. El conocimiento acerca de algunas de las características de los fármacos anticoagulantes y su mejor aplicación clínica debe redundar, indiscutiblemente, en una mejoría en la atención médica de los pacientes en el sector salud en México.

\section{Conflicto de intereses}

Los autores declaran no tener conflicto de intereses alguno.

\section{Financiamiento}

La presente investigación no ha recibido ayuda específica proveniente de agencias del sector público, sector comercial o entidades sin ánimo de lucro.

\section{Responsabilidades éticas}

Protección de personas y animales. Los autores declaran que para esta investigación no se han realizado experimentos en seres humanos ni en animales.

Confidencialidad de los datos. Los autores declaran que en este artículo no aparecen datos de pacientes.

Derecho a la privacidad y consentimiento informado. Los autores declaran que en este artículo no aparecen datos de pacientes.

\section{Bibliografía}

1. Chen T, Wu D, Chen H, Yan W, Yang D, Chen G, et al. Clinical characteristics of 113 deceased patients with coronavirus disease 2019: Retrospective Study. BMJ. 2020;368.

2. Guan W, Ni Z, Hu Y, Liang W, Ou C, He J, et al. Clinical characteristics of coronavirus disease 2019 in China. N Engl J Med. 2020;30;38:1708-20.

3. Wang D, Hu B, Hu C, Zhu F, Liu X, Zhang J, et al. Clinical characteristics of 138 hospitalized patients with 2019 novel coronavirus-infected pneumonia in Wuhan, China. JAMA. 2020;323:1061-9. 
4. Zhou F, Yu T, Du R, Fan G, Liu Y, Liu Z, et al. Clinical course and risk factors for mortality of adult in patients with COVID-19 in Wuhan, China: A retrospective cohort study. Lancet. 2020;395:1054-62.

5. Huang C, Wang Y, Li X, Ren L, Zhao J, Hu Y, et al. Clinical features of patients infected with 2019 novel coronavirus in Wuhan, China. Lancet. 2020;395:497-506.

6. Lippi G, Favaloro EJ. D-dimer is associated with severity of coronavirus disease 2019: A pooled analysis. Thromb Haemost. 2020;120:876-8.

7. Thachil J, Tang N, Gando S, Falanga A, Cattaneo M, Levi M, et al. ISTH interim guidance on recognition and management of coagulopathy in COVID-19. J Thromb Haemost. 2020;18:1023-26.

8. Klok F, Kruip M, van der Meer N, Arbous S, Gommers FAMPJ, Kant M, et al. Incidence of thrombotic complications in critically ill ICU patients with COVID-19. Thromb Res. 2000;191:145-7

9. Llitjos J-F, Leclerc M, Chochois C, Monsallier JM, Ramakers M, Auvray M et al. High incidence of venous thromboembolic events in anticoagulated severe COVID-19 patients. J Thromb Haemost. 2000;18:1743-6.

10. Tang N, Li D, Wang X, Sun Z. Abnormal coagulation parameters are associated with poor prognosis in patients with novel coronavirus pneumonia. J Thromb Haemost. 2020;18:844-7.

11. Castelli R, Gidaro A. Abnormal hemostatic parameters and risk of thromboembolism among patients with COVID-19 infection. J Hematol. 2020; $9 \cdot 1-4$

12. Tang N, Bai H, Chen X, Gong J, Li D, Sun Z. Anticoagulant treatment is associated with decreased mortality in severe coronavirus disease 2019 patients with coagulopathy. J Thromb Haemost. 2020:18:1094-9.

13. Paranjpe I, Fuster V, Lala A, Russak A, Glicksberg BS, Levin MA, et al. Association of treatment dose anticoagulation with in-hospital survival among hospitalized patients with COVID-19. JACC. 2020;76:122-4.

14. Poissy J, Goutay J, Caplan M, Parmentier E, Duburcq, Lassalle F, et al. Pulmonary embolism in COVID-19 patients: Awareness of an increased prevalence. Circulation. 2020;142:184-6.
15. Wang J, Hajizadeh N, Moore EE, Mclntyre RC, Moore PK, Veress LA et al. Tissue plasminogen activator (tPA) treatment for COVID-19 associated acute respiratory distress syndrome (ARDS): A case series. J Thromb Haemost. 2000;18:1752-5.

16. Middeldorp S, Coppens $M$, van Haaps TF, Foppen M, Vlaar AP Müller MCA, et al. Incidence of venous thromboembolism in hospitalized patients with COVID-19. J Thromb Haemost. 2020;18:1995-2002.

17. Bikdeli B, Madhavan MV, Jimenez D, Chuich T, Dreyfus I, Driggin E, et al. COVID-19 and thrombotic or thromboembolic disease: Implications for prevention, antithrombotic therapy, and follow-up. JACC. 2000;75:2550-73.

18. Connors JM, Levy JH. COVID-19 and its implications for thrombosis and anticoagulation. Blood. 2020;135:2033-44.

19. Leisman DE, Deutschman CS, Legrand M. Facing COVID-19 in ICU: vascular dysfunction, thrombosis, and dysregulated inflammation. Intensive Care Med. 2020;46:1105-8.

20. Zhai Z, Li C, Chen C, Gerotziafas G, Zhang Z, Wan J, et al. Prevention and treatment of venous thromboembolism associated with coronavirus disease 2019 infection: A consensus statement before guidelines. Thromb Haemost. 2020;120:937-48.

21. Song JC, Wang G, Zhang W, Zhang Y, Li WQ, Zhou Z. Chinese expert consensus on diagnosis and treatment of coagulation dysfunction in COVID-19. Mil Med Res. 2020;7:19.

22. COVID-19 Treatment Guidelines Panel. Coronavirus Disease 2019 (COVID-19) Treatment Guidelines [Internet]. National Institutes of Health [acceso: 31 mayo 2020]. Disponible en: https://www.covid19treatmentguidelines.nih.gov/

23. Spyropoulos AC, Lipardi C, Xu J, Peluso C, Spiro TE, De Sanctis $Y$, et al. Modified IMPROVE VTE Risk score and elevated D-dimer identify a high venous thromboembolism risk in acutely ill medical population for extended thromboprophylaxis. TH Open. 2020;4:e59-e65.

24. Cohen AT, Harrington RA, Goldhaber SZ, Hull RD, Wiens BL, Gold A, et al. Extended thromboprophylaxis with betrixaban in acutely ill medical patients. N Engl J Med. 2016;375:534-44 